\title{
Science-based wildlife disease response
}

In 2007, the current outbreak of African swine fever (ASF), which severely affects wild boar populations and pigs, reached the Caucasus region. Since then, the virus has spread into eastern Europe and some places in central and western Europe (such as Belgium) through wild boar, domestic pigs, and human activities. The virus has raised serious concerns in countries with large pork industries, which may suffer economic losses due to trade restrictions. To control the outbreak, national authorities have taken drastic but likely ineffective measures that disregard the science of wildlife management. Poland, for example, has massively increased culling of wild boar to minimize ASF spread and the risk of transmission to domestic pigs, despite opposition by experts. The policy does not include population monitoring that could evaluate its effectiveness. It also does not limit wild boar access to agricultural crops and game feed, which is a key driver of population growth. Meanwhile, Denmark is building a $70-\mathrm{km}$ border fence to exclude cross-border migration of wild boar. The fence will disrupt wildlife habitats, but it will not stop the virus from spreading through the transportation of live pigs, wild boar, or pig- and wild boar-derived tissues and products or through the movement of other objects carrying the virus, such as human clothing. Factors that govern wild boar abundance and virus spread are not bound by national borders. Instead of haphazard policies, we urge governments to agree on a coordinated response that adheres to the principles of modern wildlife management. Adaptive wildlife management strategies consider the human dimension and prevent unsound reactive management. Improved wildlife population monitoring and analysis are the best ways to determine which approaches to wildlife management are successful ecologically, economically, and socially. Sustainable management will depend on local circumstances and national wildlife management regulations, but science-based strategies can be implemented at the continental scale. Legislators across Europe should consult scientists and wildlife and animal health agencies before making decisions about wildlife policy. European countries should coordinate population monitoring and management. Shared responsibility for wildlife management among countries will enable funding for research that can critically evaluate its success. The ASF crisis can serve as a chance to develop a science-based wildlife policy for Europe.

Joaquín Vicente, Marco Apollonio, Jose A. Blanco-Aguiar, Tomasz Borowik, Francesca Brivio, Jim Casaer, Simon Croft, Göran Ericsson, Ezio Ferroglio, Dolores Gavier-Widen, Christian Gortázar, Patrick A. Jansen, Oliver Keuling, Rafał Kowalczyk, Karolina Petrovic, Radim PIhal, Tomasz Podgórski, Marie Sange, Massimo Scandura, Krzysztof Schmidt, Graham C. Smith, Ramon Soriguer, Hans-Hermann Thulke, Stefania Zanet, Pelayo Acevedo. 